Eur. J. Clin. Chem. Clin. Biochem.

Vol. 30, 1992, pp. 809-814

(C) 1992 Walter de Gruyter \& Co. Berlin · New York

\title{
Significance of Bone Alkaline Phosphatase, CA 15-3 and CEA in the Detection of Bone Metastases During the Follow-Up of Patients Suffering from Breast Carcinoma
}

\author{
By Petra Stieber ${ }^{1}$, Dorothea Nagel ${ }^{1}$, Christiane Ritzke ${ }^{1}$, N. Rössler ${ }^{1}$, C. M. Kirsch ${ }^{3}$, W. Eiermann ${ }^{2}$ and \\ A. Fateh-Moghadam ${ }^{1}$ \\ 1 Institut für Klinische Chemie \\ 2 Abteilung für Gynaekologie und Geburtshilfe \\ ${ }^{3}$ Abteilung für Nuklearmedizin der radiologischen Klinik \\ Klinikum Großhadern, Ludwig-Maximilians-Universität, München, Germany
}

(Received February 21/July 30, 1992)

Summary: After the introduction $(1,2)$ and methodical evaluation $(3,4)$ of a new method for the quantitative measurement of the bone isoenzyme of alkaline phosphatase (test-combination bone alkaline phosphatase, Boehringer Mannheim), we started a retrospective clinical study for the follow-up investigations of breast cancer patients. Our aim was to establish the significance of the routinely used tumour markers, CEA and CA 15-3, in combination with bone alkaline phosphatase for the early detection of metastatic spread to the bone. We investigated 492 sera from 92 patients suffering from breast carcinoma, and we compared each date of investigation with the results of the clinical examination and with the results of medical imaging, if that had been performed. From a previous study involving skeleton scintigraphy (5) we knew that single examinations do not allow a differential diagnosis between benign and malignant disorders of the bone, so we based our calculations on differences between sequential investigations. We found that in follow-up investigations of patients with breast carcinoma the combined determination of CEA, CA 15-3 and bone alkaline phosphatase may be indicative for the localisation of metastatic disease. The determination of the bone alkaline phosphatase is easy to handle with a short assay time and good reproducibility; it can therefore be recommended.

\section{Introduction}

Breast cancer is still the most frequent malignant disease in women, with growing incidence and mortality rates in Western countries. In the follow-up of these tumour patients the early detection of relapse or metastases is of great importance for successfull treatment and therefore influences prognosis. The determination of tumour markers in serum, like CEA and CA $15-3$, is useless for screening or primary diagnosis of breast carcinoma, but has been found by many investigators to be helpful for monitoring the course of disease and development of relapse. Although blood markers have an adjunctive role for the

1) Enzyme: alkaline phosphatase, EC 3.1.3.1. earlier diagnosis of recurrent disease, they are not able to discriminate between the localization of the metastatic spread. Knowing the low specificity and sensitivity of skeleton scintigraphy for the early detection of bone metastases and the high risk of the development of bone involvement in patients suffering from breast carcinoma, it would be of great interest to determine whether the joint assay of bone alkaline phosphatase $^{1}$ ) and tumour markers would provide an aid to the localization of the metastatic spread. For this purpose we determined CA 15-3, CEA, total alkaline phosphatase and bone alkaline phosphatase in the sera of breast cancer patients and compared our results with the clinical course, skeleton scintigraphy and $\mathrm{X}$-ray diagnostics. 


\section{Patients}

The present retrospective study was done with frozen sera stored at $-70^{\circ} \mathrm{C}$ in our serum bank. We investigated sera from 92 patients with breast carcinoma. At least three blood samples and the results of corresponding clinical investigations had to be available for inclusion of a patient in the study.

The number of investigations was distributed as follows:

\begin{tabular}{ccc}
\hline & $\begin{array}{l}\text { Number of } \\
\text { investigations }\end{array}$ & $\begin{array}{l}\text { Number of } \\
\text { patients }\end{array}$ \\
\hline 3 & 15 \\
& 4 & 17 \\
& 5 & 21 \\
& 6 & 19 \\
& 7 & 9 \\
& 8 & 8 \\
& 11 & 1 \\
& 13 & 1 \\
\hline Total & 492 & 92 \\
\hline
\end{tabular}

The intervals between two consecutive investigations varied between 6 days (minimum) and 1085 days (maximum). The mean interval was 207 days, the median 160 days.

Of the 400 investigations with existing preceding values we decided not to take into account the last date of investigation (no confirmed results), in order to guarantee reliable clinical data. In the same way all investigations with a vague clinical background or any kind of uncertain and later unconfirmed results did not enter the subsequent calculations. Therefore the number of investigations finally entering the evaluation was significantly lower than mentioned above under "number of investigations", and the grouping of patients and pairs of investigations was as follows:

1) first investigation revealed no bone metastases

a) no suspicion of bone metastases during the whole followup $(\mathrm{n}=89)$

b) first investigation revealed no bone metastases, but bone metastases developed at some time during the follow-up. In this case the first investigation included in the study was the last one without bone metastases $(n=30)$

2) first investigation with bone metastases
a) regressive follow-up $(n=10)$
b) stable follow-up $(n=8)$
c) progressive follow-up $(n=37)$

Each patient was likely to be included more than once in the subsequent calculations. With the exception of group 1a), all patients were likely to change their group during the investigation (patients with regressive disease in the beginning developed progressive disease during the evaluation and vice versa).

\section{Methods}

\section{Clinical chemistry}

\section{Alkaline phosphatase}

Total alkaline phosphatase was determined according to the guidelines of the German Society of Clinical Chemistry, on the Hitachi 717 at $25^{\circ} \mathrm{C}$ (inter-assay CV: $5 \%$ ).

\section{Bone alkaline phosphatase}

Bone alkaline phosphatase was determined according to the method of Rosalki \& Foo (1) by precipitation with wheat germ lectin (test combination BAP, Boehringer Mannheim; interassay CV: $10 \%$ ).

\section{CA $15-3$}

The cancer antigen CA 15-3 was determined by an automated enzyme immunoassay based upon two monoclonal antibodies 115-D8 and DF 3 (Enzymun, Boehringer Mannheim; interassay CV: $5 \%$ ).

\section{Carcinoembryonic antigen}

The carcinoembryonic antigen (CEA) was determined by an automated microparticle enzyme immunoassay (MEIA) based upon two monoclonal antibodies (IMx, Abbott; inter-assay CV: $10 \%)$.

\section{Clinical and physical examination}

At each stage (date) of the investigation we compared the results of the four markers in blood with the results of the clinical examination, and with the results of medical imaging (if available), i.e. skeleton scintigraphy, X-ray, or computer tomography.

\section{Definition of "bone metastases"}

Proof of bone metastases was obtained by skeleton scintigraphy, $\mathrm{X}$-ray of the bone or computer tomography. In cases of contradictory findings the stronger and more precise result was taken (for instance a positive X-ray is "stronger" than a negative skeleton scintigraphy, and a positive scintigraphy is "stronger" than a negative X-ray. Both of these are more significant than an unsuspicious clinical investigation).

\section{Statistics}

\section{Differences between consecutive investigations}

In a first step the differences (absolute and relative) of the four markers bone alkaline phosphatase, total alkaline phosphatase, CEA and CA 15-3 between two consecutive times of investigation were calculated.

The entire evaluation was based on these differences between two consecutive investigations and not punctual dates of investigation.

After determining the absolute and relative differences between the single investigations we calculated mean values, standard deviations, medians, percentiles, specificity and sensitivity. The significances were tested using the Wilcoxon test

\section{Results}

\section{Distribution of differences between two con-} secutive investigations

Figure 1 shows the distribution of the differences of values for bone alkaline phosphatase. The median of the differences for group 1a) is $1 \mathrm{U} / 1$, whereas in group 1b) (development of bone metastases) it was $10 \mathrm{U} / \mathrm{l}$. 


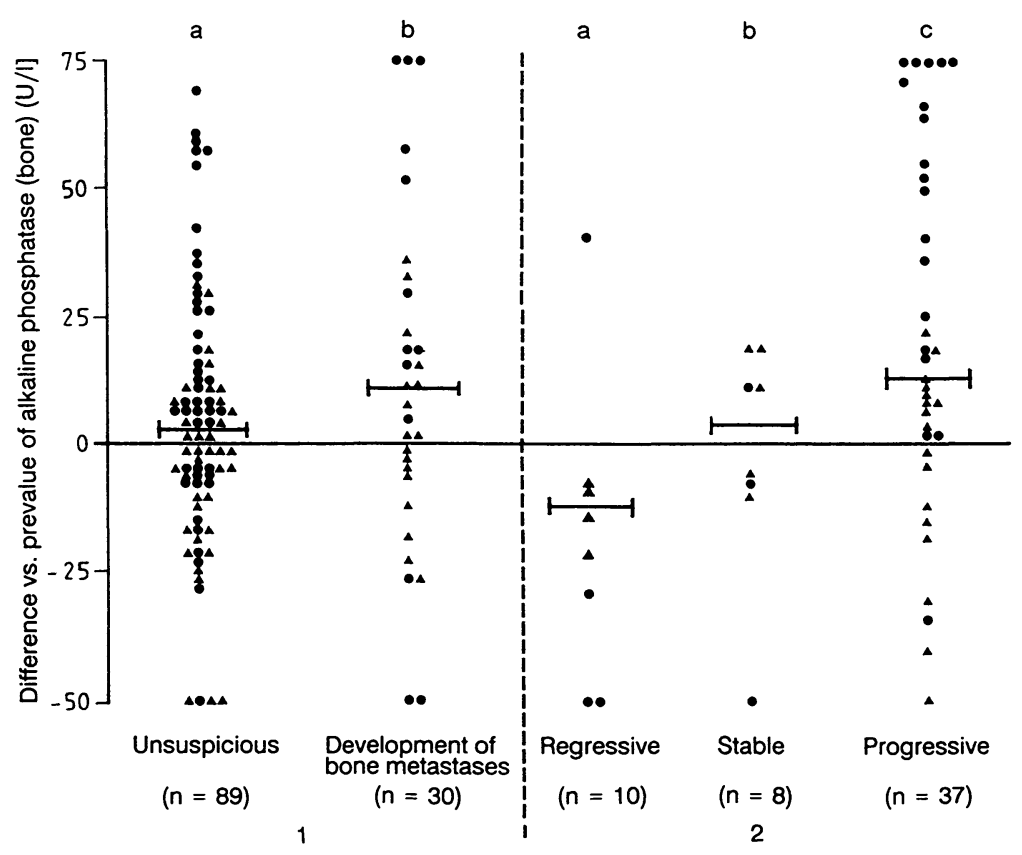

Fig. 1. Distribution of the differences between consecutive values of bone alkaline phosphatase for the 5 groups (from left to right):

1a) unsuspicious follow-up,

1b) development of bone metastases,

2c) progressive follow-up.

When the second value was outside the reference range the difference is plotted as a dot. When the second value was inside the reference range $(\leq 70 \mathrm{U} / \mathrm{l})$ the difference is plotted as a triangle.

Group 1a) (bone metastases but regressive disease) showed a negative median $(-12 \mathrm{U} / \mathrm{l})$ of values corresponding to decreasing values from one investigation to the other. Group 2c) (progressive disease) revealed a median of $12 \mathrm{U} / \mathrm{l}$ (increasing values).

The distribution of the absolute values of the 5 groups of pairs revealed that group $1 \mathrm{a}$ and $2 \mathrm{~b}$, as well as group $1 b$ and $2 c$, could be taken together. The lack of a significant difference between the groups taken together was confirmed by the Wilcoxon test. Further calculations were done for the following 2 groups of investigations:

Group $A(\mathrm{n}=97)$ : Continuous follow-up without bone metastases (1a) and stable disease (2b).

Group $B(\mathrm{n}=67)$ : Development of bone metastases at some time during the investigation (1b) and progressive disease (2c).

The number of investigations with regressive disease (2a) was to small to give statistically reliable results.

As expected for group B, the values of all 4 markers investigated were significantly higher than those for group A. But also within group A (unsuspicious and stable follow-up) we observed very large differences between two consecutive values. These differences were especially marked for the tumour markers CA $15-3\left(363 \cdot 10^{3} \mathrm{U} / \mathrm{l}\right)$ and CEA $(54 \mu \mathrm{g} / \mathrm{l})$, which is about 15 times the reference range; they were less pronounced for bone alkaline phosphatase $(70 \mathrm{U} / \mathrm{l})$ and alkaline phosphatase $(104 \mathrm{U} / \mathrm{l}$, within the reference range) (tab. 1).

Very similar results were obtained for relative differences from the preceding values (data not shown in this publication). However, relative differences have the drawback of overestimating changes in the range of low values.

Specificity, sensitivity, positive and negative predictive value

To investigate the efficiency of a marker in detecting bone metastases or progressive follow-up, suitable sensitivities had to be calculated. Thus, sensitivities were defined as the number of investigations with a significant increase of the marker compared with the preceding value, divided by the number of all pairs investigated in group B (progressive disease). Analogously, specificity was defined by the proportion of those pairs with clinically unsuspicious follow-up (group A) that showed no significant increase in value.

A significant increase was defined as a change of value of more than 2 times the CV (corresponding to a significant change with a probability of about $70 \%$ ). 
Tab. 1. Differences between consecutive values of bone alkaline phosphatase, alkaline phosphatase, CA 15-3 and CEA for group A (stable follow-up) and group B (progressive disease and detection of bone metastases).

\begin{tabular}{|c|c|c|c|c|c|c|c|c|}
\hline \multirow[b]{3}{*}{ Group of patients } & \multicolumn{8}{|c|}{ Difference from the prevalue (preceding investigation) } \\
\hline & \multicolumn{2}{|c|}{$\begin{array}{l}\text { Bone } \\
\text { alkaline } \\
\text { phosphatase } \\
(\mathrm{U} / \mathrm{l})\end{array}$} & \multicolumn{2}{|c|}{$\begin{array}{l}\text { Total } \\
\text { alkaline } \\
\text { phosphatase } \\
(\mathrm{U} / \mathrm{l})\end{array}$} & \multicolumn{2}{|c|}{$\begin{array}{l}\text { CA } 15-3 \\
\left(10^{3} \mathrm{U} / 1\right)\end{array}$} & \multicolumn{2}{|c|}{$\begin{array}{l}\text { CEA } \\
(\mu \mathrm{g} / \mathrm{l})\end{array}$} \\
\hline & A & B & A & B & A & B & A & $\mathrm{B}$ \\
\hline Mean & 3 & 20 & 10 & 47 & 7 & 46 & 1 & 7 \\
\hline Median & 2 & 11 & 9 & 27 & 1 & 6 & 0 & 1 \\
\hline $25^{\text {th }}$ percentile & -8 & -5 & -10 & 0 & -2 & 0 & 0 & 0 \\
\hline $75^{\text {th }}$ percentile & 14 & 37 & 27 & 76 & 4 & 32 & 1 & 3 \\
\hline Max. decrease & -90 & -61 & -91 & -144 & -97 & -188 & -6 & -84 \\
\hline Max. increase & 70 & 305 & 104 & 393 & 363 & 1404 & 54 & 169 \\
\hline Valid $\mathrm{N}$ & 97 & 67 & 97 & 67 & 97 & 67 & 97 & 67 \\
\hline
\end{tabular}

Tab. 2. Specificity, sensitivity, positive and negative predictive values of CA 15-3, CEA, alkaline phosphatase and bone alkaline phosphatase in the detection of bone metastases, based on an increase of at least 2 times the coefficient of variation.

\begin{tabular}{lllllc}
\hline Marker & & $\begin{array}{l}\text { Bone alkaline } \\
\text { phosphatase }\end{array}$ & $\begin{array}{l}\text { Total alkaline } \\
\text { phosphatase }\end{array}$ & CA 15-3 & CEA \\
\hline Specificity & $(\%)$ & 70 & 58 & 62 & 66 \\
Sensitivity & $(\%)$ & 52 & 67 & 63 & 54 \\
PV + (prevalence 68\%) & $(\%)$ & 79 & 77 & 78 & 77 \\
PV + (prevalence 2\%) & $(\%)$ & 3 & 2 & 4 & 3 \\
PV - (prevalence 68\%) & $(\%)$ & 41 & 45 & 44 & 40 \\
PV - (prevalence 2\%) & $(\%)$ & 99 & 99 & 99 & 98 \\
\hline
\end{tabular}

We obtained the highest specificity $(70 \%)$ for bone alkaline phosphatase and the lowest $(58 \%)$ for total alkaline phosphatase (due to the fact that alkaline phosphatase consists of several isoenzymes, which cause many "false positive" results). Conversely, the bone isoenzyme of alkaline phosphatase revealed the lowest percentage of true positive results $(52 \%)$, while total alkaline phosphatase ( $67 \%$ sensitivity) revealed the highest percentage (tab. 2).

In addition to specificity and sensitivity, we calculated the positive and negative predictive values. For this purpose, the prevalence of progression of metastatic disease had to be assumed. From our data we assumed a probability of $68 \%$ for progression of disease within group 2c), so that all 4 markers attained a high positive predictive value between 77 and $79 \%$ (tab. 2).

The probability that the patients without bone metastases (group 1b) would develop involvement of the bone before the next date of examination was assumed to be $2 \%$, and the corresponding positive predictive values varied between 2 and $4 \%$.

\section{Combination of 2 or 3 markers}

The aim of the study was to enable a localization of the metastatic spread by combining the tumour markers CEA and CA 15-3 with bone alkaline phosphatase. We therefore restricted the subsequent calculations to combinations of markers that are relevant to this question: CEA and CA 15-3 on the one hand, and bone alkaline phosphatase on the other hand. Both markers had to fulfil an increase of at least two times the coefficient of variation. Therefore the number of false positive results decreases (specificity between 81 and $91 \%$ !) and sensitivity is obviously lower (between 27 and $32 \%$ ) (tab. 3).

In combining the 3 markers, we set the necessary increase for bone alkaline phosphatase at 2 times the $\mathrm{CV}$, while at least one of the tumour markers (CEA or CA 15-3) had to fulfil the same increase.

Under these requirements, specificity decreases a little to $81 \%$, and the proportion of true positive results increases to $41 \%$. 
Tab. 3. Specificity, sensitivity, positive and negative predictive values for combinations of bone alkaline phosphatase, alkaline phosphatase, CA 15-3 and CEA in the detection of bone metastases from breast carcinoma. In each case both markers have to increase by at least 2 times the coefficient of variation.

\begin{tabular}{|c|c|c|c|c|}
\hline Marker & $\begin{array}{l}\text { Specificity } \\
(\%)\end{array}$ & $\begin{array}{l}\text { Sensitivity } \\
(\%)\end{array}$ & $\begin{array}{l}\mathrm{PV}+ \\
(\%)\end{array}$ & $\begin{array}{l}\mathrm{PV}- \\
(\%)\end{array}$ \\
\hline CEA + Bone alkaline phosphatase & 91 & 27 & 86 & 37 \\
\hline $\begin{array}{l}\text { CA } 15-3 \text { + Bone alkaline phosphatase } \\
\text { CEA + Bone alkaline phosphatase }\end{array}$ & 87 & 32 & 84 & 42 \\
\hline or $15-3+$ Bone alkaline phosphatase & 81 & 41 & 82 & 40 \\
\hline
\end{tabular}

Tab. 4. Postulation: $95 \%$ specificity of CEA, CA $15-3$ and bone alkaline phosphatase and the corresponding required increases of the sensitivities and the positive and negative predictive values of the markers.

\begin{tabular}{lllll}
\hline Marker & $\begin{array}{l}\text { Increase } \\
(\%)\end{array}$ & $\begin{array}{l}\text { Sensitivity } \\
(\%)\end{array}$ & $\begin{array}{l}\text { PV }+ \\
(\%)\end{array}$ & $\begin{array}{l}\text { PV - } \\
(\%)\end{array}$ \\
\hline CEA & 250 & 15 & 86 & 45 \\
CA 15-3 & 100 & 21 & 90 & 36 \\
Bone alkaline phosphatase & 90 & 21 & 90 & 36 \\
Total alkaline phosphatase & 60 & 28 & 92 & 38 \\
CEA + Bone alkaline phosphatase & $39 / 30$ & 23 & 91 & 37 \\
CA 15-3 + Bone alkaline phosphatase & $25 / 30$ & 25 & 94 & \\
CEA + Bone alkaline phosphatase & $50 / 30$ & 37 & & 42 \\
or & $18 / 30$ & & & \\
CA 15-3 + Bone alkaline phosphatase & & & & \\
\hline
\end{tabular}

\section{Claim: Overall specificity of $95 \%$}

In order to be able to compare the clinical significance of the different markers we fixed the specificity for all single marker determinations and their combinations at $95 \%$ (tab. 4).

In order to reach this high fraction of true negative results the increase of the marker from the first determination to the second had to be very clear - for example $250 \%$ for CEA and $90 \%$ for bone alkaline phosphatase.

Naturally, the sensitivity decreases: $15 \%$ for CEA, and $21 \%$ for CA $15-3$ and for bone alkaline phosphatase. A combination of tumour markers and bone alkaline phosphatase produces a slight increase of sensitivity (25\% for CA $15-3$ and bone alkaline phosphatase).

When either CEA or CA 15-3 is combined with bone alkaline phosphatase the increase of value necessary to attain a specificity of $95 \%$ has to be significantly lower: $50 \%$ for CEA, $18 \%$ for CA $15-3$ and $30 \%$ for bone alkaline phosphatase. The proportion of true positive results rises to $37 \%$ and the positive predictive value is $94 \%$.

Thus, when CEA and/or CA 15-3 is combined with the determination of bone alkaline phosphatase, the increase of values necessary to demonstrate development of metastatic spread to the bone is relatively low compared with the single determination (tab. 4).

\section{Early detection of bone metastases}

Of the 60 patients who started the present investigation without bone metastases, 30 developed bone in involvement at some time during the study. Eleven of these patients were not submitted to any sort of therapy during development of bone metastases. Three patients showed slightly decreased values of bone alkaline phosphatase while developing bone metastases, the bone alkaline phosphatase concentration of one patient was stable, and 7 follow-ups showed increasing values.

This effect becomes clearer when the tumour markers CEA and CA 15-3 are combined with bone alkaline phosphatase. Figure 2 demonstrates the parallel and obvious increase of CEA and bone alkaline phosphatase with development of bone metastases.

\section{Discussion}

Although metastases are not usually found at the initial diagnosis of breast carcinoma, local and distant recurrence of disease is very frequent. Meanwhile it 


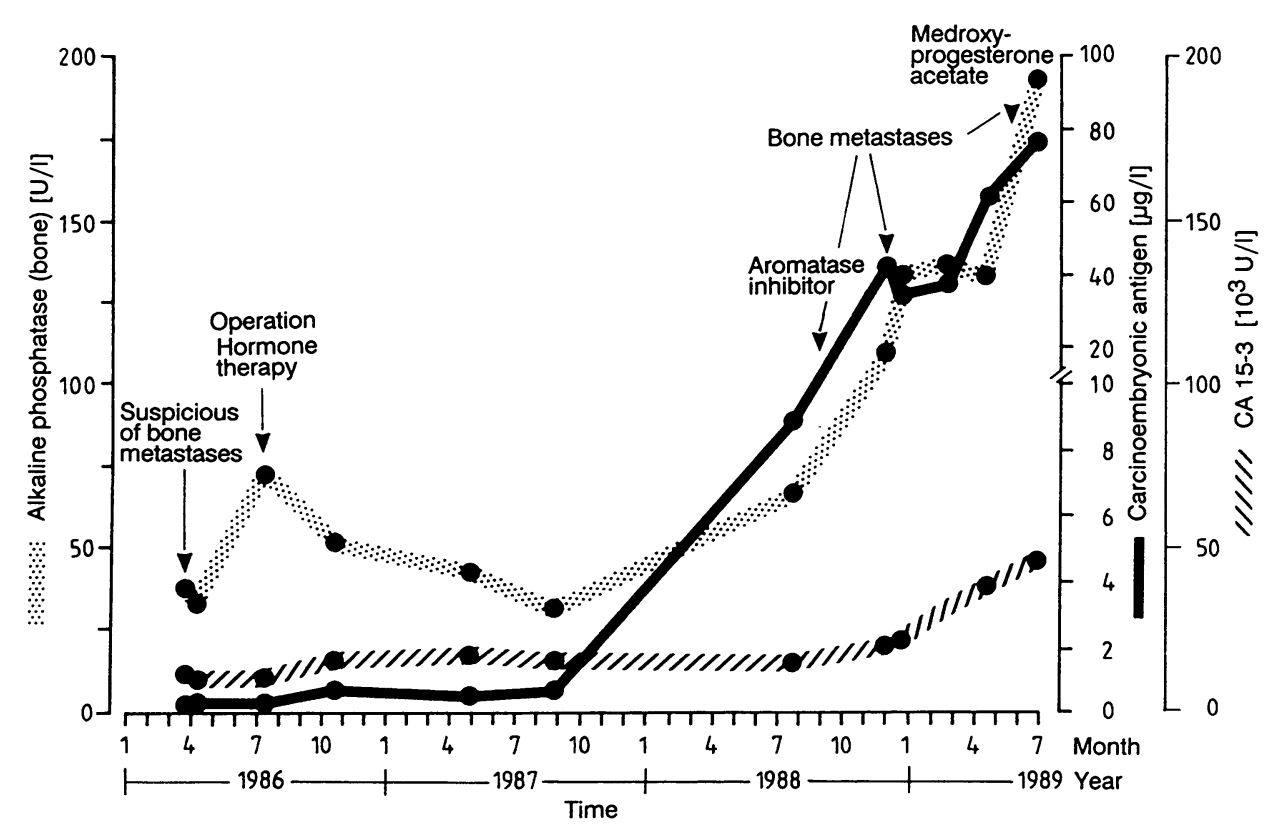

Fig. 2. Follow-up of serum CEA, CA 15-3 and bone alkaline phosphatase in a patient with breast carcinoma metastatic to the bone. (BA. E. ? 73a, operated March 27, $1986 \mathrm{~T}_{3} \mathrm{~N}_{2} \mathrm{M}_{\mathbf{x}}$ ) There is an obvious parallel increase of CEA and bone alkaline phosphatase with the development of bone metastases.

has been proven by many investigators that blood markers like CA 15-3 and CEA in breast cancer can play an important role in the early detection of metastatic spread. When determined after primary surgery during the follow-up of breast cancer patients they often increase and reveal progressive disease long before it is manifested clinically. But even if tumour markers indicate a progression of disease, the localization of metastatic spread represents a great problem, in view of the low specificity and sensitivity of bone scanning and $\mathrm{X}$-ray investigations.

From our results it is evident that progression of the disease is detected very early by the combined determination, and, moreover, that it is very probably (depending on the extent of increase) located in bone. For these reasons, but also from a methodical (the quantitative determination of bone alkaline phosphatase is easy to handle with good reproducibility) and an economic (determination of bone alkaline phosphatase is not expensive but comparable to alkaline phosphatase) standpoint, the combined determination of bone alkaline phosphatase, CEA and CA 15-3 for follow-up investigations of breast cancer patients can be recommended. We will continue this study in order to include a greater number of patients, but also to investigate the role of total alkaline phosphatase, bone alkaline phosphatase and $\gamma$-glutamyltransferase in the localization and differential diagnosis of metastatic spread to the liver and the bone.

\section{References}

1. Rosalki, S. B. \& Foo, A. Y. (1984) Two new methods for separating and quantifying bone and liver alkaline phosphatase isoenzymes in plasma. Clin. Chem. 30, 1182-1186.

2. Klein, G., Bodenmüller, H. \& Gerber, M. (1989) Evaluation of a new photometric assay for the determination of human bone alkaline phosphatase. Clin. Chem. 35, 1089.

3. Falkner, Ch., Stieber, P., Mraz, W., Neumeier, D. \& FatehMoghadam, A. (1989) Untersuchungen zur Bestimmung der Knochenform der alkalischen Phosphatase im Serum durch Präzipitation mit Weizenkeimlektin. J. Clin. Chem. Clin. Biochem. 27, 767-768.

4. Rosalki, S. B., Foo, A. Y., Burlina, A., Prellwitz, W., Stieber, P., Neumeier, D., Klein, G., Poppe, W. \& Bodenmüller, H. (1992) Multicenter evaluation of a test kit (Iso-alp) for the measurement of bone alkaline phosphatase in plasma. Clin. Chem. Accepted, has to be corrected.
5. Stieber, P., Kirsch, C. M., Nagel, D., Falkner, Ch., Roessler, N., Neumeier, D. \& Fateh-Moghadam, A. Stellenwert der alkalischen Knochenphosphatase und alkalischen Gesamtphosphatase im Vergleich mit der Skelettszintigraphie in der Detektion von Knochenmetastasen. Wird eingereicht in Clin. Chem.

6. Lamerz, R., Reithmeier, A., Stieber, P., Eiermann, W. \& Fateh-Moghadam, A. (1991) Role of blood markers in the detection of metastases from primary breast cancer. Diagn. Oncol. 1, 88-97.
Dr. Petra Stieber
Institut für Klinische Chemie
Klinikum Großhadern
Marchioninistraße 15
W-8000 München 70
Bundesrepublik Deutschland 